\title{
Tree Structured Model of Skin Lesion Growth Pattern via Color Based Cluster Analysis
}

\author{
Sina KhakAbi ${ }^{1,2,3}$, Tim K. Lee ${ }^{1,2,3}$, and M. Stella Atkins ${ }^{1,2}$ \\ 1 School of Computing Science, Simon Fraser University, Canada \\ $\{$ skhakabi, stella\}@sfu.ca \\ 2 Department of Dermatology and Skin Science, University of British Columbia and \\ Vancouver Coastal Health Research Institute, Canada \\ 3 Cancer Control Research Program, BC Cancer Research Centre, Canada \\ tlee@bccrc.ca
}

\begin{abstract}
This paper presents a novel approach to analysis and classification of skin lesions based on their growth pattern. Our method constructs a tree structure for every lesion by repeatedly subdividing the image into sub-images using color based clustering. In this method, segmentation which is a challenging task is not required. The obtained multi-scale tree structure provides a framework that allows us to extract a variety of features, based on the appearance of the tree structure or sub-images corresponding to nodes of the tree. Preliminary features (the number of nodes, leaves, and depth of the tree, and 9 compactness indices of the dark spots represented by the sub-images associated with each node of the tree) are used to train a supervised learning algorithm. Results show the strength of the method in classifying lesions into malignant and benign classes. We achieved Precision of 0.855 , Recall of 0.849 , and F-measure of 0.834 using 3-layer perceptron and Precision of 0.829, Recall of 0.832, and F-measure of 0.817 using AdaBoost on a dataset containing 112 malignant and 298 benign lesion dermoscopic images.
\end{abstract}

\section{Introduction}

Malignant melanoma is one of the most frequent types of cancer in the world. In recent decades, the annual rate of its incidence has been increasing by $3 \%-7 \%$ in fair-skinned populations [12. It is increasing faster than any other cancers in the world, and in 2008, melanoma was the sixth most common malignancy in men and the seventh in women [6]. Despite the fact of lethality of skin cancer, if the malignant lesion is detected early, it can be cured without complication. Hence, there is a growing demand for the computer-aided-diagnosis of melanoma to improve the diagnostic accuracy.

There are several different modalities to obtain images of skin lesions. One reliable way to screen skin lesions is to use a dermoscope [4, a non-invasive hand-held device which captures magnified digital skin images using either polarized light or oil immersion to render the outermost layer of the skin, called 


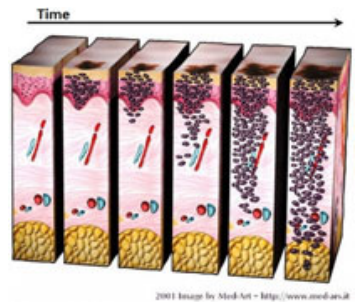

Fig. 1. The vertical growth phase of pigmented skin lesion over time. Melanocytes penetrate into dermis and change the coloration of the lesion. (c) 2001 by Med-Art http://www.med-ars.it)

the epidermis, translucent. This enables dermatologists to recognize the subsurface structures that are associated with malignancy. Therefore, image processing algorithms can be incorporated to facilitate the diagnosis.

For the conventional use of dermoscopy without computer assistance, dermatologists have proposed different scoring methods that facilitate the diagnostic process. The following methods are mainly used for this purpose [2]: 1) $A B C D$ rule; 2)pattern analysis; 3)Menzie's method; 4) 7-point checklist; and 5)texture analysis. Every method highlights specific features which are biologically inspired, arising from the structure of malignant lesions. Features extracted using these methods are mainly based on shape structure, texture and color variation. Recently, many studies have been carried out for extracting the diagnostic features using image processing techniques. In [11, an overview of some of these techniques is provided.

In this study, a novel approach inspired by the analysis of the growth pattern of skin lesions is proposed. A malignant lesion is often identified with two growth phases, radial and vertical [5]. Both malignant and benign lesions start growing radially. In this phase, pigmented lesion is formed by nests of melanocytes, which synthesize a brown pigmentation called melanin. This phase happens in the epidermis. Invasive melanoma forms in the vertical phase when malignant melanocytes start penetrating into dermis (see Fig. 10. In this phase, an invasive melanoma tends to show multiple colors due to the position of melanin in different skin layers, formation of blood vessels, and regression of the lesion. Melanin is dark brown in epidermis, tan near the dermoepidermal junction, and blue-gray in the dermis. This is caused by different absorbance of light in different layers of skin. Accordingly, pigmented malignant and benign lesions demonstrate a radial growth pattern which can be observed using dermoscopy. This pattern is also used in [13] as a spatial constraint to achieve a better segmentation of the lesion.

We use a multi-scale tree structure for analyzing the growth pattern of lesions. This framework allows us to study: i) radial growth pattern of the individual dark spots, and ii) distribution of the dark spots over the lesion. In section 2 , the method for constructing the tree structure is explained. This structure provides a strong framework to extract various features. In section 3, some preliminary features are discussed to demonstrate the strength of the provided framework. Section 4 describes the implementation in detail. Section 5 presents performance evaluation of the method based on the features extracted in section 3 . Section 6 concludes the paper. 


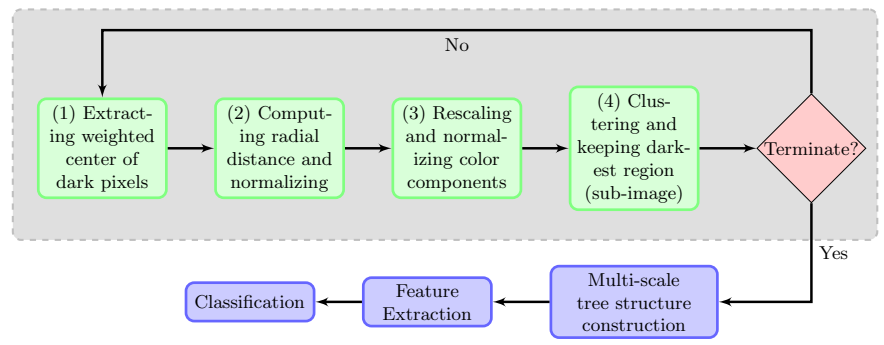

Fig. 2. Flowchart of the provided algorithm

\section{Method}

We study two main features based on the coloration of the lesion. Our novel method takes a top-down approach in which every skin lesion image is repeatedly sub-divided into sub-images through a clustering procedure over the pixels. At every iteration of the sub-division, the color features and a spatial coordinate feature (see sub-section 2.2) of all pixels in the sub-image are rescaled and clustered into 2 clusters. The darkest cluster of pixels is retained and formed into a new sub-image. The process of rescaling and reclustering is repeated for the new sub-images until termination. In other words, we are constructing a multiscale tree structure such that every sub-image corresponds to a node in the tree and its children are obtained by rescaling the image features, reclustering pixels, and retaining the darker cluster. Fig. 2 demonstrates the schematic view of our algorithm and Fig. 3 illustrates the results of applying the algorithm.

As a preprocessing step, we removed dark hairs using Dullrazor [9]. The following sub-sections explain the algorithm in detail towards creation of the multiscale tree structure. Feature extraction and classification steps are discussed later.

\subsection{Extraction of Weighted Center of Dark Pixels}

For every iteration, RGB image is converted to gray-scale image using the following equation

$$
I=0.2989 \times R+0.5870 \times G+0.1140 \times B
$$

then $I$ is normalized between 0 and 1 and darker pixels with $I \leq I_{d}$ are extracted. As a result, $X$ and $Y$ which are two sets of coordinates, are defined as follows

$$
X=\left\{x \mid I_{x, y} \leq I_{d}\right\} \text { and } Y=\left\{y \mid I_{x, y} \leq I_{d}\right\}
$$

Then, $\left(x_{C}, y_{C}\right)$ the coordinates of the center point are calculated as follows

$$
x_{C}=\frac{1}{|X|} \times \sum_{i=1}^{|X|} x_{i} \text { and } y_{C}=\frac{1}{|Y|} \times \sum_{i=1}^{|Y|} y_{i}
$$

Simply, $x_{C}$ and $y_{C}$ are the centroid of the dark pixels. 


\subsection{Computing Radial Distance}

The radial distance $D_{x, y}$ is computed for every pixel in the current sub-image by calculating the Euclidean distance between pixels and the extracted center, where

$$
D_{x, y}=\sqrt{\left(x-x_{C}\right)^{2}+\left(y-y_{C}\right)^{2}}
$$

Consequently, all the pixels on the same circle centered on the extracted center point, will have the same radial distance. The goal is to include this distance as a feature in the clustering stage in next step. This will lead to layered clusters of pixels which represent the growth pattern of the spot centered on the point that is the weighted average over the dark pixels.

\subsection{Clustering and Shrinking}

Incorporating the radial distance from the center of an image as a spatial constraint in clustering pixels is provided in [13] to facilitate skin lesion segmentation. We modified and extended this idea. Instead of clustering around the center of an image, we cluster around the centroid of a dark spot. In addition, we extend the method to multi-scale tree structure. In every iteration, we have a sub-set of pixels of the original image. This sub-set is clustered into two clusters using the well-known $k$-means clustering algorithm. In the clustering stage, a four-dimensional normalized feature set, containing three color components and the previously computed radial distance, is fed to the algorithm with different weights assigned. We chose Hue-Saturation-Intensity (HSI) color space to build the feature set. Therefore, RGB color space is converted to HSI color space using equations given in [7]. Thus, for pixel $(x, y)$ the feature set is $\left\{H_{x, y}, S_{x, y}, I_{x, y}, D_{x, y}\right\}$. After obtaining two clusters, the darker cluster is characterized and kept by looking for the highest $I$ value in the resulting clusters' color-map. The new sub-set of pixels in the dark cluster delimits regions over the image. These regions undergo hole filling and opening morphological operations and define our final sub-images. Every sub-image obtained so far will be entered in the loop independently and all aforementioned three steps will be repeated for them and for their descendant sub-images. This is where the idea of the tree structure arises.

Sub-image decomposition ends when one of these four conditions is true: $i$ ) number of pixels in the obtained sub-image is less than a constant value, ii) color variance over the blue and green intensity channels in a sub-image is less than a threshold, iii) descendant of a sub-image has not significantly changed in comparison to its parent, or $i v$ ) the depth of the implicitly constructed tree is more than a limit. Further details are provided in section 4.

\subsection{Multi-scale Tree Structure Construction}

The above process can be interpreted as a depth first search (DFS) over a tree. When a sub-image breaks into multiple sub-images, each sub-image is traversed 


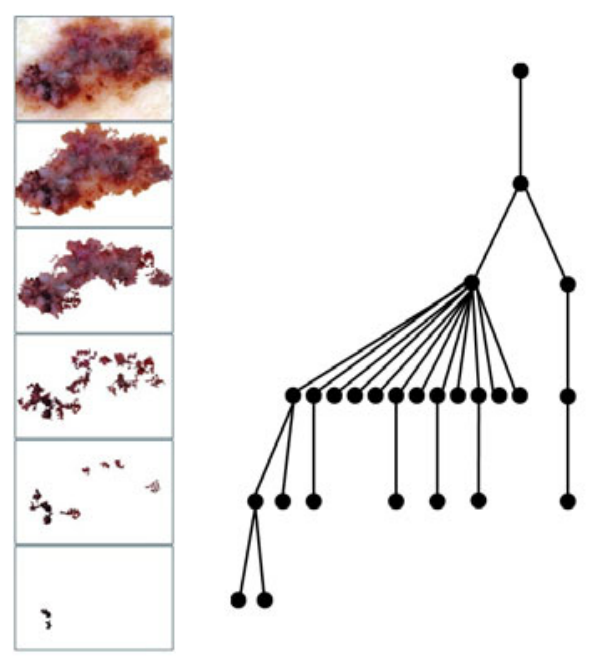

(a) (b)

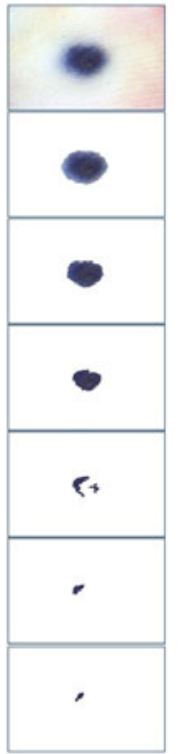

(c)

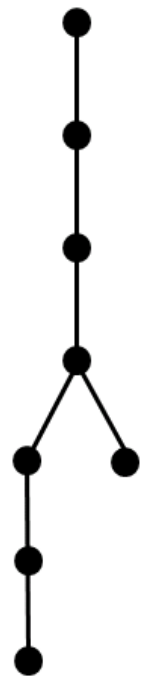

$(d)$

Fig. 3. (a) and (c) illustrate decomposition of a malignant and benign lesions respectively. Their corresponding tree structures are also shown in (b) and (d). Number of branches and leaves in the benign lesion is less than that of malignant lesion.

until termination, then the next sub-image is traversed and so on. Hence, the main image corresponds to the root of the tree; its sub-images form its children in the second layer; the third layer consists of sub-images of the second layer; and so forth. Fig. 3 illustrates samples of the extracted tree structure, for a benign and a malignant lesion.

Our hypothesis is that if the complexity of the tree structure is high, the probability of malignancy is also high. Complexity can be defined as the number of nodes and the degree at every node. This can be seen in examples provided in Fig. 3 .

\section{Feature Extraction}

The tree structure framework allows us to extract a variety of features, based on the tree itself or the sub-images at each node of the tree. In this study some preliminary features are examined to illustrate the strength of the framework. The number of nodes and leaves in the tree and depth of the tree are very basic features that can be used in a classification procedure. As can be seen in Fig. 3 , these features reflect the complexity of the tree structure to some extent. Compactness index is chosen as a sub-image based feature which reflects the irregularity of the border of the sub-image. It is calculated using as follows 


$$
C I=\frac{P^{2}}{4 \pi A}
$$

where $P$ is the perimeter of the object and $A$ is the object area. The extracted compactness index is stored in an array, where element $i$ of the array corresponds to depth $i+1$ of the tree. The $C I$ is calculated for all the nodes in depth $i$ and is summed up and multiplied by $\frac{10-i}{9}$ which is proportional to the depth. Borders of sub-images which are placed close to the root of the tree are more similar to the border of the lesion in the main image. As a result, they are more reliable and their corresponding nodes should be given more weight than nodes close to leaves. The results of using these features separately are discussed in section 5. The $C I$ is not calculated for the root as it is the main image with square border. Thus, the length of the array should be one less than the maximum possible depth of the tree which is defined in the loop termination conditions. Other features such as border irregularity index [10] can be extracted and used instead of $C I$; however, their time cost is higher than calculating $C I$.

\section{Implementation Detail}

We implemented the algorithm using the MATLAB's image processing toolbox. In this study, thresholds and parameters of the algorithm are experimentally chosen, as the algorithm is not very sensitive to these values. Extracting the center point as the first step in every iteration of the loop, is not sensitive to the threshold chosen for the gray-scale image intensities. This threshold $\left(I_{d}\right)$ is set to 0.25 . Increasing this value will cause incorporating of more pixels over the image and change the center point slightly. This change will affect the value of $D_{x, y}$. As the number of clusters in the clustering stage is set to two, we are assured that the darker cluster includes all the dark regions that we are interested in. Consequently, the final tree structure will have the same shape. This is an advantage of our algorithm.

The weights assigned to the H, S, and I are set to 2 and the weight of radial distance $D$ is set to 1 in the clustering stage. Higher weighting on $D$ results in rounded borders of sub-images in the nodes close to the leaves. The low weighting on $D$ and higher weighting on color components reduce the sensitivity to the threshold value for center extraction step.

Values of parameters and thresholds for termination conditions are as follows: i) area of sub-images should be more than 500 pixels, ii) the variance over the both blue and green channels should be greater than 40 when the blue and green channel values are normalized between 0 and 255 , iii) difference in area between two consecutive nodes should be more than 50 pixels, and $i v$ ) the maximum allowed depth is set to 10 . 


\section{Results}

We prepared a dataset of 410 images taken from [1. These images were picked out of 763 images to provide sufficient malignant lesions in the data set for machine learning algorithms. This was done without visual inspection and only by looking at the diagnosis of lesions. In this dataset, we have 112 malignant lesion images (containing melanoma and basal cell carcinoma (BCC)), and 298 benign lesion images (containing congenital, compound, dermal, Clark, spitz, and blue nevus; dermatofibroma; and seborrheic keratosis). Our ground truth is based on the information provided in [1. This information is based on dermatologists' diagnosis. WEKA [8] is used to classify the images. 3-layer perceptron and AdaBoost are chosen as classifiers. The parameters for 3-layer perceptron are set as follows: learning rate is set to 0.3 , momentum is set to 0.2 , training time is set to 500 and validation threshold is set to 20. The parameters of AdaBoost are set as follows: the number of iterations is set to 10, the seed is randomly generated and the weight threshold is set to 100.

All the features which are computed in the feature extraction section are gathered in a 12-dimensional feature set (number of nodes, number of leaves, depth, and 9 CI components) and the resulting set is fed into the classifiers. Validation method is set to leave-one-out ten-fold cross validation. The malignant and benign images are randomly chosen and uniformly distributed over the folds. Table 1 illustrates the classification results between malignant and benign classes for our dataset using two different classifiers. In this table, we also provide the result of the classification using Betta et al.'s streaks detection technique [3], which we reimplemented in MATLAB and evaluated using the same dataset. In Betta's method, the image is subdivided into 16 equal rectangular blocks and, like our method, the dark brown color is examined in HSI color space, though Betta uses it for detecting streaks. However, Betta's method is very sensitive to the segmentation of the lesion.

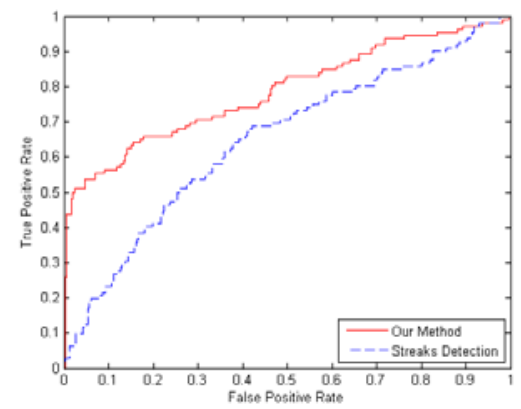

(a)

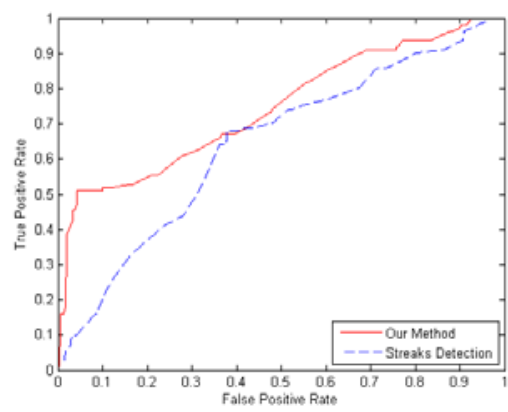

(b)

Fig. 4. Receiver Operating Characteristic (ROC) curve of classification based on our method and streaks detection method [3] using (a) 3-layer preceptron classifier and (b) AdaBoost classifier. The areas under the curves are provided in Table 1. 
Table 1. Table of results of classifying the dataset into malignant and benign classes using 12 features

\begin{tabular}{|c|c|c|c|c|c|}
\hline Method & Classifier & Precision & Recall & F-Measure & AUC of ROC \\
\hline Our Method & 3-layer perceptron & 0.855 & 0.849 & 0.834 & 0.786 \\
\hline Streaks Detection & 3-layer perceptron & 0.689 & 0.732 & 0.656 & 0.648 \\
\hline Our Method & AdaBoost & 0.829 & 0.832 & 0.817 & 0.745 \\
\hline Streaks Detection & AdaBoost & 0.639 & 0.724 & 0.619 & 0.642 \\
\hline
\end{tabular}

Table 2. Results of classifying the data-set using $C I$ in different layers of the tree

\begin{tabular}{|l|c|c|c|c|c|}
\hline Feature set & Classifier & Precision & Recall & F-Measure & AUC of ROC \\
\hline CI1 to CI3 & 3-layer perceptron & 0.639 & 0.712 & 0.641 & 0.617 \\
\hline CI4 to CI9 & 3-layer perceptron & 0.713 & 0.729 & 0.622 & 0.494 \\
\hline CI1 to CI3 & AdaBoost & 0.692 & 0.732 & 0.685 & 0.637 \\
\hline CI4 to CI9 & AdaBoost & 0.596 & 0.722 & 0.614 & 0.490 \\
\hline
\end{tabular}

The values reported for Precision, Recall, and F-measure are weighted average values based on the size of the class. Fig. 4 represents the ROC curve of the classifications for two methods and two different classifiers. Our method outperformed streaks detection method using both classifiers as shown in Table 1 and Fig. 4.

We also studied the effect of $C I$ alone in lower and higher nodes in the extracted tree. Table 2 provides the results of this study using the AdaBoost and the 3-layer preceptron classifier and justifies using higher weights for nodes close to root.

\section{Conclusion}

We have developed a multi-scale tree structure based iterative framework for feature extraction and classification of the skin lesions. The strength of this framework is that it does not require an explicit lesion segmentation, which could be a challenging problem. As illustrated in this paper, we build a structure based on the distribution of dark spots; however, we could also build a tree structure with light color spots or regions with special characteristics and textures. We also demonstrated that a good classification result was achieved using just a few preliminary features.

We will further expand the features to include color, texture and other shape information. The optimal parameters of the algorithm could be learnt using an optimization procedure. Finally, we will validate our method on other large data sets.

Acknowledgements. This research was funded by NSERC and CIHR. The authors would also like to thank to Dr. Harvey Lui and Dr. David McLean for their guidance. 


\section{References}

1. Argenziano, G., Soyer, H.P., et al.: Interactive Atlas of Dermoscopy (Book and CD-ROM). Edra Medical Publishing and New Media (2000)

2. Argenziano, G., Soyer, H.P., et al.: Dermoscopy of pigmented skin lesions: Results of a consensus meeting via the internet. Journal of the American Academy of Dermatology 48(5), 679-693 (2003)

3. Betta, G., Di Leo, G., Fabbrocini, G., Paolillo, A., Scalvenzi, M.: Automated application of the 7-point checklist diagnosis method for skin lesions: Estimation of chromatic and shape parameters. In: IEEE Instrumentation and Measurement Technology Conference, vol. 3, pp. 1818-1822 (2005)

4. Binder, M., Schwarz, M., et al.: Epiluminescence microscopy: A useful tool for the diagnosis of pigmented skin lesions for formally trained dermatologists. Arch. Dermatol. 131(3), 286-291 (1995)

5. Clark, W.H., Ainsworth, A.M., Bernardino, E.A., Yang, C.H., Mihm, C.M., Reed, R.J.: The developmental biology of primary human malignant melanomas. Semin. Oncol. 2(1), 83-103 (1975)

6. Erickson, C., Driscoll, M.S.: Melanoma epidemic: Facts and controversies. Clinics in Dermatology 28(3), 281-286 (2010)

7. Gonzalez, R.C., Woods, R.E.: Digital Image Processing, 2nd edn. Addison-Wesley Longman Publishing Co., Inc., Amsterdam (2001)

8. Hall, M., Frank, E., Holmes, G., Pfahringer, B., Reutemann, P., Witten, I.H.: The weka data mining software: an update. SIGKDD Explor. Newsl. 11, 10-18 (2009)

9. Lee, T., Ng, V., Gallagher, R., Coldman, A., McLean, D.: Dullrazor §: A software approach to hair removal from images. Computers in Biology and Medicine 27(6), 533-543 (1997)

10. Lee, T.K., McLean, D.I., Atkins, M.S.: Irregularity index: A new border irregularity measure for cutaneous melanocytic lesions. Medical Image Analysis 7(1), 47-64 (2003)

11. Maglogiannis, I., Doukas, C.: Overview of advanced computer vision systems for skin lesions characterization. IEEE Transactions on Information Technology in Biomedicine 13(5), 721-733 (2009)

12. Marks, R.: Epidemiology of melanoma. Clinical and Experimental Dermatology $25(6), 459-463(2000)$

13. Zhou, H., Chen, M., Zou, L., Gass, R., Ferris, L., Drogowski, L., Rehg, J.: Spatially constrained segmentation of dermoscopy images. In: 5th IEEE International Symposium on Biomedical Imaging: From Nano to Macro, ISBI 2008, pp. 800-803 (May 2008) 